\title{
ESTUDO ANTROPOMÉTRICO DE PRÉ- ESCOLARES DA CALHA DO RIO NEGRO, AMAZONAS, BRASIL. II- BARCELOS
}

\author{
Fernando Helio ALENCAR ${ }^{1}$, Lúcia Kiyoko O. YUYAMA ${ }^{1}$, Dionísia \\ NAGAHAMA ${ }^{1}$, Rosana, C. P. PARENTE ${ }^{2}$
}

RESUMO - Foi realizado no município de Barcelos (1995) estudo transversal, onde avaliouse o estado nutricional de 240 pré- escolares da área urbana e 43 na área rural, por meio de avaliação antropométrica, adotando-se a classificação de Gomez e os critérios propostos pela OMS. Constatou-se pela classificação de Gomez, que $41,7 \%$ das crianças da área urbana apresentavam algum grau de desnutrição, predominando a forma leve- (DI) $32,1 \%$, acometendo indistintamente todas as faixas etárias, sendo de apenas $2,5 \%$ a ocorrência da forma grave (DIII). Na área rural, o quadro registrado não difere muito quanto a intensidade $(37,2 \%)$, contudo, mostra-se mais atenuado em relação a gravidade da desnutrição. Segundo os critérios da OMS, $31,1 \%$ das crianças do municipio apresentavam inadequação no indicador "altura/idade" (desnutrição crônica), constatando-se uma relativa proteção nas crianças no primeiro ano de vida $(10,2 \%)$. A inadequação no indicador "peso/altura" (desnutrição aguda), foi constatada em apenas $4,2 \%$ dos pré-escolares do município, acometendo principalmente as crianças no primeiro ano de vida. Estes resultados evidenciam as precárias condições de saúde e nutrição da referida população.

Palavras-chaves: Antropometria; Estado Nutricional; Desnutrição Aguda; Nanismo Nutricional.

Antropometric Study of Pre-Echool Children in the Rio Negro Valey, Amazonas State, Brazil. II-Barcelos

ABSTRACT - A cross-sectional study was carried out in the municipal district of Barcelos (Amazonas State, Brazil) in 1995, involving 240 pre-school children in the urban area and 43 in the interior, in order to evaluate their nutritional status according to antropometric indicators. According to the Gomez classification, in the urban area $41,7 \%$ of the children presented some degree of malnutrition, with the mild form DI predominanting $(32,1 \%)$ and affecting all agegroups indiscrimanately; the prevalence of the severe form DIII was $2,5 \%$. In the interior $37,2 \%$ of the children presented some degree of malnutrition, but the prevalence of the more severe forms was lower. According to the criteria of the WHO, 31,1\% of the children presented a height for age ratio indicative of chronic undernourishiment, less frequent in the first year of life $(10,2 \%)$. Weight for height ratios indicative of acute undernutrition were detected in $4,2 \%$ of the sample, mainly in children under 12 months old. These results are an indication of the precarious state of health and nutrition of the study population.

Keys-words: Antropometry; Nutritional Status; Acute Malnutrition; Nutritional Stunting.

\section{INTRODUÇÃO}

Quando se analisa o conjunto dos problemas de saúde que afetam as populações dos paises em desenvolvimento, a Desnutrição Protéico-Energética (DPE), ainda figura como um dos principais problemas da saúde pública e de abrangência social (OMS, 1993; OPAS, 1994; UNICEF,1995).
Ao longo dos últimos anos, muitos pesquisadores têm enfatizado a gravidade do problema nutricional para alguns países da América Latina, onde, além da deficiência de proteína e energia, são freqüentes as carências de vitamina A, riboflavina, ferro e iodo. (Gandra,1970; Cook et al.,1971; Lechting \& Arroyave, 1979; Ariza Macias et al., 1980; OPAS, 1994; Unicef, 1995).

Instituto Nacional de Pesquisa da Amazonia

2 Departamento de Estatística da Universidade do Amazonas 
No Brasil, há uma vasta literatura mostrando que são cinco as endemias carenciais, configurados há muito tempo, como verdadeiros problemas de saúde pública: Desnutrição Proteico-Energética (DPE), Anemia ferropriva, Hipovitaminose A, Cárie dental e Bócio endêmico. Obviamente, a população do Norte e Nordeste contribuiu mais, significativamente, para o dimensionamento deste quadro nosológico, onde são assinaladas as prevalências mais elevadas de DPE. (João et al., 1979; Giugliano et al., 1981; Giugliano et al., 1984; Araújo, 1988; Vannucchi et al., 1992).

Em nosso meio, estudos das condições de vida, saúde e nutrição, abrangendo os diferentes segmentos $\mathrm{e}$ grupos populacionais da Amazônia, são relativamente escassos, alguns dos quais realizados há mais de duas décadas. Mais frequentemente estas pesquisas referem- se, principalmente, à população infantil de Manaus, sendo raros e fragmentados os estudos enfocando as condições de saúde, nutrição, sobrevivência e qualidade de vida do homem no contexto rural Amazônico. Não obstante, os dados referidos nestes trabalhos, ainda representam informações válidas e importantes subsídios ao entendimento do processo Saúde/ Doença e carências nutricionais que afetam as populações da região Norte do Brasil. A análise evolutiva dos trabalhos enfocando as condições de Saúde e Nutrição realizados no Amazonas nestas duas últimas décadas, revela um perfil pluricarencial em alguns grupos populacionais, caracterizado por: baixa ingestão de zinco, tiamina, riboflavina, vitamina A, cálcio, calorias, elevado percentual de déficit pônderoestatural, baixo peso ao nascer e elevada ocorrência de parasitas gastroentestinais (Pinheiro et al., 1976; Shrimpton \& Giugliano, 1979; Giugliano et al., 1984; Shrimpton, 1984; Lehti, 1989; Marinho, 1988; Yuyama et al., 1989; Nagahama et al., 1990; Alencar et al., 1991; Ferraroni et al., 1991; Marinho et al., 1992).

Considerando a carência de informações atualizadas sobre as condições de saúde de grupos populacionais da Amazônia, o presente trabalho objetiva, primordialmente, contribuir para a composição do diagnóstico dos processos carenciais das populações dos diferentes ecossistemas Amazônicos : Rio Negro, Amazonas, Solimões, Madeira e Purus.

\section{Casuística e Métodos}

\section{Local:}

Barcelos é o maior município do estado do Amazonas, com uma área de $89572 \mathrm{~km}^{2}$ e uma população de 10856 habitantes, o que configura uma densidade demográfica de $0,12 \mathrm{hab} /$ $\mathrm{km}^{2}$. Situa-se na margem direita do Rio Negro, afluente do Rio Amazonas, a $40 \mathrm{~m}$ acima do nivel do mar, distando da capital do estado, $396 \mathrm{~km}$ em linha reta e $496 \mathrm{~km}$ por via fluvial. Localiza- se ao Norte do Estado do Amazonas, limitando-se com os municípios de Novo Airão, Codajás, Moura, Santa Isabel do Rio Negro, com o estado de Roraima e República da Venezuela a $0^{\circ} 58^{\prime} 01^{\prime \prime}$ de latitude Sul e $62^{\circ} 56^{\prime} 00^{\prime \prime}$ de longitude a Oeste de Greenwich (SEBRAE- Am/1994). 


\section{Universo:}

O presente trabalho objetivou estudar as crianças pré- escolares, residentes no município de Barcelos. Para isto realizamos um cadastramento populacional (busca ativa) em todos os bairros e nas localidades de maior densidade demográfica, sendo incluidos no estudo, exclusivamente, as familias que apresentavam crianças em idade igual e/ ou inferior a 5 anos.

Obedecendo estes critérios foram registradas 230 familias, compreendendo um contingente populacional de 1189 pessoas, com uma média de 5,2 pessoas por familia. $\mathrm{O}$ universo de estudo ficou, inicialmente, constituido por 312 pré- escolares (presentes no momento da entrevista). No entanto, obedecendo os normas estabelecidas pelo Software EPINUT(Epi/Info - 6.0), quanto ao cálculo exato da idade, foram excluidas 29 crianças ( $>60$ meses), ficando o universo estudado assim definido: 240 pré- escolares da área urbana (bairros de Aparecida, São Lázaro, Nazaré, São Sebastião) e 43 da área rural (nas localidades de São Luiz, Moura, Baturité, Cumarú e São Domingos).

\section{Instrumentos de Pesquisa e seus critérios de Medida}

\section{Estado Nutricional:}

Na avaliação do estado nutricional dos pré- escolares, empregamos, inicialmente, a classificação de Gomez(1946), que utiliza o indicador "peso/ idade" em função do sexo e classifica o estado nutricional em porcentagem de adequação: Normal (> 90\%); desnutridos do grau I ( $90 \%$ a $75 \%$ ); grau
II $(75 \%-60 \%)$ e grau III $(<60 \%)$. Na diferenciação dos tipos de desnutrição, utilizamos os critérios propostos pela OMS que considera como limite discriminatório de desnutrição, desvios correspondentes a população de referência inferior a " $<-2$ Escores Z" para os indicadores : "altura/ idade"(desnutrição pregressa) e peso/ altura"(desnutrição aguda); sendo o "escore- Z" igual ao número de unidade de desvios- padrão entre a medida observada e o seu respectivo valor esperado da população de referencia. O cálculo das adequaçôes teve como referencial o padrão proposto pelo "National Center for Health Statistcs - NCHS (1977).

\section{Análise Estatística}

Para a análise dos dados foram utilizados os testes de comparação de duas proporções, e o Qui-quadrado de Pearson, com nivel significância de $5 \%$, obtidos pelo programa: Measure/ Epi- Info-6.0).

\section{RESULTADOS}

\section{Avaliação Nutricional}

A Tabela 1 mostra as medidas de prevalência da desnutrição, para os diferentes critérios de avaliação. Segundo a classificação de Gomez, $41,0 \%$ dos pré- escolares do município de Barcelos, apresentavam algum grau de desnutrição: $31,1 \%$ na forma leve (DI), $7,8 \%$ na moderada (DII) e $2,1 \%$ na forma grave (DIII). Em relação a faixa etária, a desnutrição incide, indistintamente em todas as crianças, porém com menor intensidade nas mais jovens $(31,3 \%$ no primeiro ano), tendendo a se intensificar em função da idade 
Tabela 1. Prevalência da Desnutrição Proteico-Energética em Pré-Escolares, segundo alguns critérios de Avaliação Nutricional. Municipio de Barcelos- AM (1995).

\begin{tabular}{lcccccc}
\hline \multirow{2}{*}{$\begin{array}{l}\text { Indicador } \\
\text { Antropométrico }\end{array}$} & \multicolumn{2}{c}{ ÁREA } & \multicolumn{2}{c}{$\begin{array}{c}\text { ESTUDADA } \\
\text { Rural }\end{array}$} & \multicolumn{2}{c}{ Município } \\
\cline { 2 - 8 } & No & $\%$ & No & $\%$ & No & $\%$ \\
\hline Gomez & 140 & $(58,3)$ & 27 & $(62,8)$ & 167 & $(59,0)$ \\
Eutrofia & 77 & $(32,1)$ & 11 & $(25,6)$ & 88 & $(31,1)$ \\
DI & 17 & $(7,1)$ & 5 & $(11,6)$ & 22 & $(7,8)$ \\
DII & 6 & $(2,5)$ & - & $(-)$ & 6 & $(2,1)$ \\
DIII & 77 & $(32,2)$ & 11 & $(25,6)$ & 88 & $(31,1)$ \\
OMS (<- 2 Escores Z) & 11 & $(4,5)$ & 1 & $(2,3)$ & 12 & $(4,2)$ \\
Altura/ Idade(*) & & & & & & \\
Peso/ Altura(**) & 240 & $(100,0)$ & 43 & $(100,0)$ & 283 & $(100,0)$ \\
\hline Crianças Estudadas & & & & & &
\end{tabular}

(*) Urbano x Rural $p=0,198$

("*) Urbano $\times$ Rural $p=0,249$

(49,1\% nas crianças de 49 a 60 meses) (Tab.2). Os bairros de maior ocorrência de Desnutrição EnergéticoProtéica (DEP) foram: Nazaré $(46,1 \%)$ e São Lázaro $(44,8 \%)$ e dentre as localidades rurais estudadas, as que apresentaram maior inadequação nutricional foram: Baturité $(60,0 \%)$ e São Luiz (50,0\%) (Tab. 3).

$\mathrm{Na}$ diferenciação dos tipos de desnutrição, constatamos que $31,1 \%$ das crianças do Municipio apresen- taram inadequação no indicador "altura/ idade", registrando- se menor acometimento nas crianças mais jovens $(10,2 \%$ no primeiro ano de vida); a partir desta idade, constata-se maior frequencia de crianças com desnutrição crônica. A inadequação no indicador "peso/ altura", foi constatada em apenas $4,2 \%$ dos préescolares do município; acometendo principalmente as crianças no primeiro ano de vida. (Tabs. 2, 4).

Tabela 2. Identificação do grupo de risco, quanto a ocorrência de Desnutrição Protéico-Energética (Gomez), em pré- escolares do município de Barcelos- Am (1995).

\begin{tabular}{|c|c|c|c|c|c|c|c|c|c|c|c|c|}
\hline Municipio & \multicolumn{6}{|c|}{ Estado Nutricional (Gomez) } & & & & & & \\
\hline \multicolumn{13}{|l|}{ Idade } \\
\hline \multirow[t]{2}{*}{ (meses) } & \multicolumn{2}{|c|}{ Eutróficos } & \multicolumn{2}{|c|}{ Leve } & \multicolumn{2}{|c|}{ Moderada } & \multicolumn{2}{|c|}{ Grave } & \multicolumn{2}{|c|}{ Desnutridos } & \multicolumn{2}{|c|}{ Geral } \\
\hline & $\mathrm{N}^{2}$ & $\%$ & $N^{0}$ & $\%$ & $\mathrm{~N}^{2}$ & $\%$ & $\mathrm{~N}^{2}$ & $\%$ & $\mathrm{~N}^{2}$ & $\%$ & $\mathrm{~N}^{0}$ & $\%$ \\
\hline $0-12$ & 35 & 68,6 & 7 & 13,7 & 5 & 9,8 & 4 & 7,8 & 16 & 31,3 & 51 & 100,0 \\
\hline $13-24$ & 42 & 59,1 & 23 & 32,3 & 4 & 5,6 & 2 & 2,8 & 29 & 40,7 & 71 & 100,0 \\
\hline 25. 36 & 27 & 62,8 & 11 & 25,6 & 5 & 11,6 & 0 & 0,0 & 16 & 37,2 & 43 & 100,0 \\
\hline 37- 48 & 33 & 55,9 & 20 & 33,9 & 6 & 10,2 & 0 & 0,0 & 26 & 44,1 & 59 & 100,0 \\
\hline 49. 60 & 30 & 50,8 & 27 & 45,7 & 2 & 3,4 & 0 & 0,0 & 29 & 49,1 & 59 & 100,0 \\
\hline Total & 167 & 59,0 & 88 & 31,1 & 22 & 7,8 & 6 & 2,1 & 116 & 41,0 & 283 & 100,0 \\
\hline
\end{tabular}


Tabela 3. Identificação da localidade de risco, quanto a ocorrência de Desnutrição ProteicoEnergética (Gomez) em pré- escolares do município de Barcelos- Am (1995).

\begin{tabular}{lccccccccc}
\hline \multicolumn{1}{c}{ Universo } & Estado & \multicolumn{2}{c}{ Nutricional } \\
\hline Localidades & Estudado & \multicolumn{2}{c}{ Leve } & \multicolumn{2}{c}{ Moderada } & \multicolumn{2}{c}{ Grave } & \multicolumn{2}{c}{ Total Desnutridos } \\
\hline Área Urbana & 240 & 77 & $(32,1)$ & 17 & $(7,1)$ & 6 & $(2,5)$ & 100 & $(41,7)$ \\
\hline Aparecida & 53 & 13 & $(24,5)$ & 3 & $(5,7)$ & $2(3,8)$ & 18 & $(34,0)$ \\
S. Lázaro & 96 & 33 & $(34,4)$ & 8 & $(8,3)$ & 2 & $(2,1)$ & 43 & $(44,8)$ \\
Nazaré & 65 & 25 & $(38,4)$ & 5 & $(7,7)$ & - & 30 & $(46,1)$ \\
J. Sebastiāo & 26 & 6 & $(23,1)$ & 1 & $(3,8)$ & $2(7,7)$ & 9 & $(34,6)$ \\
\hline Área Rural & 43 & 11 & $(25,5)$ & 5 & $(11,6)$ & 0 & $(-)$ & 16 & $(37,2)$ \\
\hline Cumarú & 6 & 1 & $(16,7)$ & 1 & $(16,7)$ & - & 2 & $(33,4)$ \\
S. Luiz & 6 & 3 & $(50,0)$ & & - & - & 3 & $(50,0)$ \\
Baturité & 10 & 4 & $(40,0)$ & 2 & $(20,0)$ & - & 6 & $(60,0)$ \\
Moura & 21 & 3 & $(14,3)$ & 2 & $(9,5)$ & - & 5 & $(23,8)$ \\
\hline \multicolumn{1}{c}{ Município } & 283 & 88 & $(31,1)$ & 22 & $(7,8)$ & 6 & $(2,1)$ & 116 & $(41,0)$ \\
\hline
\end{tabular}

Urbano $\times$ Rural $p=0,292$

\section{DISCUSSÃO}

No presente trabalho o estado nutricional das crianças foi, inicialmente, definido de acordo com os critérios diagnósticos da classificação de Gomez (1946). Seguindo esta metodologia, constatamos que $41,0 \%$ dos pré- escolares do municipio de Barcelos, apresentavam inadequação no indicador "peso/idade", predominando a forma leve de desnutrição $(31,1 \%), 7,8 \%$ se enquadrando na forma moderada, sendo expressivamente baixa a ocorrência da forma grave $(2,1 \%)$. Na composição deste quadro, destaca- se a precariedade nutricional das crianças da área urbana, com $41,7 \%$ de desnutridos; sendo menos intensa $(25,6 \%)$ e grave (ausência da forma crônica), o estado nutricional das crianças da área rural. $\mathrm{Na}$ investigação da prevalência de DPE, interessou-nos também identificar o grupo de risco, visto que a ocorrência da desnutrição no primeiro ano de vida, representa maiores dificuldades para o desenvolvimento do potencial fisico e intelectual da criança, sendo, portanto, mais grave do que quando incidente em faixas etárias mais avançadas. A distribuição etária da prevalência da desnutrição nas crianças do Municipio, mostrou que ela incide, indistintamente, em todas as faixas etárias, porém com menor intensidade no primeiro ano de vida das crianças $(31,3 \%)$, e, se intensificando em função da idade ( $49,1 \%$ nas crianças com 49 a 60 meses).

Analisando estes resultados dentro do contexto histórico amazônico, encontramos na literatura o registro de maior ocorrência de desnutrição, bem como da manifestação de gravidade, ao longo das últimas décadas; por exemplo: Giugliano et al (1981), estudando a situação nutricional de pré-escolares do médio Solimões, constataram uma prevalência de $54,7 \%$ de desnutridos, com uma representação de $8,6 \%$ para as formas 
Tabela 4. Situação Nutricional de Pré-Escolares, segundo os critérios de Avaliação Nutricional da OMS, Faixa Etária e Sexo. Município de Barcelos- Am (1995).

\begin{tabular}{lcccr}
\hline & \multicolumn{5}{c}{ Critérios } & de & \multicolumn{2}{c}{ Avaliação } \\
\cline { 2 - 6 } Idade & \multicolumn{2}{c}{ Altura/ Idade $\left(^{*}\right)$} & \multicolumn{2}{c}{ Peso/ Altura $\left(^{*}\right)$} \\
\cline { 2 - 6 } Em meses & $N^{\circ}$ & $\%$ & $N^{\circ}$ & $\%$ \\
\hline OMS <-2Escores Z & & & & \\
$0-12$ & 9 & $(10,2)$ & 8 & $(66,7)$ \\
$12-24$ & 26 & $(29,5)$ & 1 & $(8,3)$ \\
$24-36$ & 11 & $(12,5)$ & 1 & $(8,3)$ \\
$36-48$ & 20 & $(22,7)$ & 2 & $(16,7)$ \\
$49-60$ & 22 & $(25,0)$ & 0 & $(-)$ \\
\hline Total & 88 & $(31,1)$ & 12 & $(4,2)$ \\
\hline Sexo & & & & \\
Masculino & 51 & $(57,9)$ & 6 & $(50,0)$ \\
Feminino & 37 & $(42,1)$ & 6 & $(5,0)$ \\
$X^{2}$ & $\mathrm{p}=$ & 12,11 & $\mathrm{p}=$ & 0,016 \\
\hline
\end{tabular}

(*) Năo foi possível o teste

moderada e grave $(p=0,0039)$. Em 1984, Giugliano et al, investigando o estado nutricional de crianças da calha do Rio Negro, relataram uma prevalência de $63,3 \%$ de desnutridos, com $15,8 \%$ das crianças se enquadrando nas formas moderada $\mathrm{e}$ grave $(p=0,00002)$. Outros autores relataram ao longo das últimas décadas, percentuais ainda superiores aos anteriormente mencionados: (Giugliano \& Shrimpton,1977; Kazapi,1989; Diniz,1991; Marinho et al, 1992). Entretanto pesquisas realizadas mais recentemente, relatam percentuais de desnutrição, expressivamente inferiores aqueles registrados há duas décadas, como referido anteriormente; por exemplo: Alencar (dados não publicados), utilizando a mesma metodologia (Gomez) e faixa etária (de 5 anos), relatam para o Rio Negro (São Gabriel da Cachoeira), um percentual de $35,6 \%$ de crianças desnutridas, predominando a forma leve $(32,8 \%)$ e de $0,9 \%$ a ocorrência da forma grave.
Para o municipio de Novo Airão, o referido autor registrou uma prevalência de $49,0 \%$ de desnutrição, sendo $38,5 \%$ na forma leve, $9,8 \%$ na moderada e $0,7 \%$ na forma grave. Da confrontação destes resultados, ressalvados obviamente as características de cada estudo, no que se refere a faixa etária analisada, pode-se admitir que a qualidade de vida, traduzida pelo estado nutricional da população infantil de Barcelos, é ainda muito precária.

Os resultados da presente pesquisa quando confrontados com aqueles da Pesquisa Nacional, sobre Saúde e Nutrição - PNSN (1990), revelam uma semelhança com os percentuais obtidos para a área urbana da região Norte; do mesmo modo, identifica para o município de Barcelos, uma situação, relativamente, mais atenuada que a do Nordeste, porém quando confrontados com as demais regiões do Brasil, percebemos o quanto ainda é precário o estado nutricional das crianças da região Norte (Tab. 5).

A classificação de Gomez ( 1946), 
utilizada neste trabalho, para definir a prevalência da desnutrição infantil, tem sido criticada, visto que, em não considerando a altura, pouca informação revelaria sobre o atraso no ganho ponderale suas causas (Chen, et al., 1980; Mata, 1983; Vasconcelos, 1993). Entretanto, sua importância epidemiológica, já é consolidada há muito tempo, tanto pelo seu poder diagnóstico, como prognóstico, na evolução dos casos graves. Neste sentido, muitas classificações, utilizando diferentes indicadores antropométricos, sucederam a classificação de Gomez; o que possibilitou um melhor entendimento da dinâmica da desnutrição (Batista Filho, 1976.; Monteiro, 1992.; Waterlow et al, 1977.; Amigo et al, 1980.; Trowbridge et al, 1980.; Vasconcelos, 1993.

Neste trabalho, para interpretarmos a gravidade da desnutrição, utilizamos também os critérios propostos pela OMS (1986), que considera a inadequação no indicador "altura/idade", como indicativo de uma situação de privação nutricional de longa duração, caracterizando assim a desnutrição pregressa (nanismo nutricional). A inadequação no indicador "peso/altura", identifica a desnutrição aguda (emaciação), refletindo um processo carencial agudo, portanto de curta duração. Assim, a utilização desta metodologia, evidenciou que $31,1 \%$ dos pré- escolares do município de Barcelos, apresentavam inadequação no indicador "altura/ idade", o que identifica um quadro de desnutrição pregressa, sendo seu acometimento, relativamente maior em crianças a partir de 1 ano de idade $(p=0,016)$. A ocorrência de desnutrição aguda ou vigente no momento da pesquisa, explicitada pela inadequação no indicador "peso/ altura", foi registrada em apenas $4,2 \%$ dos pré- escolares do Municipio, portanto sem muita relevância epidemiológica.

Não há, no contexto rural Amazônico, dados de comparação para a ocorrência de inadequação nos indicadores "altura/idade" e "peso/altura" como registrados no município de Barcelos. O presente estudo, possivelmente, é o

Tabela 5. Comparação dos dados do estudo de Barcelos (Peso/Idade) com outras pesquisas realizadas no Rio Negro, Solimões, PNSN (Norte, Nordeste, Sul e Centro- Oeste).

\begin{tabular}{|c|c|c|c|}
\hline & (n) & Desnutridos & (p) \\
\hline Barcelos $\left({ }^{*}\right)$ & 283 & 116 & 0,00002 \\
\hline Rio Negro - Giugliano et al- 1984 & 120 & 76 & \\
\hline Barcelos(*) & 283 & 116 & 0,0039 \\
\hline Rio Solimōes - Giugliano et al - 1981 & 139 & 76 & \\
\hline Barcelos $\left({ }^{*}\right)$ & 283 & 116 & 0,659 \\
\hline PNSN-Norte(Urbano) -1989 & 1020 & 432 & \\
\hline Barcelos(*) & 283 & 116 & 0,3631 \\
\hline PNSN- Nordeste - 1989 & 1020 & 432 & \\
\hline Barcelos(*) & 283 & 116 & 0,00002 \\
\hline PNSN-Sul - 1989 & 1405 & 250 & \\
\hline Barcelos $\left({ }^{*}\right)$ & 283 & & \\
\hline PNSN-Centro-Oeste-1989 & 1486 & 381 & \\
\hline
\end{tabular}


primeiro no Amazonas a utilizar como limite discriminatório entre eutrofia/ desnutrição, uma medida de dispersão traduzida em "Escores Z" para os indicadores antropométricos, "altura/ idade" e "peso/altura". O estudo de maior abrangência nacional, utilizando esta metodologia, excluiu o Norte (Rural) PNSN (1989). Areferida pesquisa apontou para a região Norte (Urbana), uma prevalência das formas crônica e aguda, respectivamente de: $23,0 \%$ e $3,1 \%$, portanto, expressivamente inferior a encontrada para os pré-escolares do Municipio de Barcelos. Para se avaliar a magnitude desta situação, há referência na literatura de que em uma população com boas condições de saúde e nutrição, espera-se encontrar apenas $2,3 \%$ de crianças com inadequação nos indicadores "altura/ idade" e "peso/altura" inferiores a "<2escores Z" (Silva et al., 1998). Deste modo o registro destes dados deixa implícito o debilitado estado geral de saúde e a grave realidade nutricional a que, historicamente, está submetida a população infantil da Região Norte. A análise dos fatores determinantes deste quadro nutricional, será objeto de outra publicação (Estudo das condições de saúde e sobrevivência da população rurral da Amazônia. III - Calha do rio Negro).

\section{Bibliografia citada}

Alencar, F.H.; Ferraroni, M.J.R.;Lehti, K.K.; Marinho, H.A.; Mota, C.S.; Silva, N.B.; Castro, J.S. 1991. Diagnóstico e perspectivas nutricionais na Região Amazônica. In: Bases Cientificas para Estratégias de Preservação e Desenvolvimento da Amazônia: Fatos e Perspectivas. Vol. I:145-154.
Amigo, H.; Begin, I.; Saenz, L.; José, A.P. 1980.Programas para extencion de cobertura en los servicios de salud; actividades de nutricion. Bol. Ofic. Sanit._Panamer., 89:480-8.

Araujo, R.I. 1988. Situação alimentar e nutriconal do Brasil. Brasília, Editora Gráfica Tipogresso.

Ariza Macias, J.; Daza, C.H.; Pradilla, A. 1980. Fundamentos de um sistema de vigilancia alimentaria nutricional. Bol.Ofic.Sanit.Panamer: 89:538-44.

Batista Filho, M., Prevalência e estágios $d a$ desnutrição proteico-calórica em crianças da cidade de São Paulo. São Paulo, 1976. 112p. (Tese - Doutorado Faculdade de Saúde Pública. Universidade de São Paulo).

Chen, L.C.; Chowdhury, A.; Huffmam, S.L. 1980. Anthropometric assessment of energy protein malnutrition and subsequent risk of mortality among pre-school age children. Amer. J. Clin. Nutr, 33:1836-45.

Cook, J.D.; Alvarado, J.; Gutniski, A.; Jamra, M.; Lambardini, J.; Linares, J.; Loria, A.; Maspes, V.; Restrepo, A.; Reynafarje, C.; Sanchez-Medal, L.; Velez, H.; Viteri, F. 1971. Nutritional deficiency and anemia in Latin America; A collabotive study. Blood, 38:591-603.

Diniz, D.B. 1991. Perfil nutricional de crianças de 2 a 6 anos de idade residentes na área urbana do municipio de Nova Olinda do Norte (AM). Dissertação de Mestrado, Universidade do Amazonas, $60 \mathrm{p}$.

Ferraroni, M. J. C.; Marinho, H.A.; Nagahama, D.; Rocha, Y.R.; Silva, N.B.; Castro, J.S. 1991. Prevalência de enteropatias na cidade de Manaus. J. Pediatria. 67:24-28.

Gandra, Y.R. 1970. La anemia ferropenica en la población de America Latina y el Caribe. Bol. Ofic. Sanit. Panamericana. 49:375-34.

Giugliano, R.; Giugliano, L.; Shrimpton, R.. 1981. Estudos nutricionais das populações rurais da Amazônia. I. Vázea do Rio Solimões. Acta Amazônica, 11:773-788.

Giugliano, R.; Shrimpton, R.; Marinho, H.A.; Giugliano, I, 1984. Estudos nutricionais das populações rurais da Amazônia. II. 
Rio Negro. Acta Amazônica, 14:427-449.

Giugliano, R.; Shrimpton, R.; 1977. Estudo antropométrico e clínico de estado nutricional em um grupo de crianças pré-escolares de Manaus. Acta Amazônica, 7:389-394.

Gomez, F. 1946. Desnutrición. Bol.Medico. Hosp.Infant. México, 3:534-51.

Instituto Nacional deAlimentação e Nutrição - INAN 1990. Pesquisa Nacional sobre Saúde e Nutrição em 1989. Perfil de Crescimento da População Brasileira de 0-25 anos. Brasilia DF, 59p.

João, W.S.J.; Brandão, M.C.; Meneses, M.A.; João, M.F.N.J, 1979. Estudo clínico nutricional em crianças pré-escolares no município de Vigia; Estado do Pará. Hiléia Médic. Belém, 1:29-34.

Kazapi, I.A.M. 1989. Estado nutricional de pré-escolares pertencentes as tres redes de ensino da cidade de Manaus (AM) em 1987. Avaliação antropométrica e do hematócrito. Dissertação de Mestrado. Universidade do Amazonas, $80 \mathrm{p}$.

Lechtig, A.; Arroyave, G, 1979. El problema nutricional em America Latina: definición, causas y lineas de acción para aliviarlo. Bol. Ofic. Sanit. Panamer. 86:478-92.

Lehti, K.K. 1989. Iron, folic acid and zinc intakes and status of pow socio-economic pregnant and lactating Amazoniam Womem. Eur. J. Clin. Nutr: 43:505-513.

Mata, L. 1983.Critérios para evaluar el estado nutricinal del niño. Gen, 37:107-23.

Marinho, H.A. 1989. Influência de parasitose intestinal (Ascaris lumbricoides e ou Giardia lambia) sobre os niveis séricos de vitamina $A$ em crianças recebendo suplementação oral de zinco elou vitamina $A$. estudo em pré-escolares de um bairro pobre de Manaus, Amazonas. Dissertação de Mestrado. Universidade do Amazonas, 86 p.

Marinho, H.A.; Kazapi, I.M.; Fernandes, H.H.J.M.; Guedes, M.R. 1992. Avaliação do estado nutricional de pré-escolaress de classe sócio-econômica média e baixa da cidade de Manaus. Estudo comparativo. Acta Amazônica, 22:363-368.
Monteiro, M.F.G.; Cervimi, R. org. Perfil estatistico de crianças e mães no Brasil; aspectos de saúde e nutrição de crianças no Brasil, 1989. Rio de janeiro, 1992.

Nagahama, D.; Marinho, H.A.; Rocha, Y.; Ferraroni, M.J.R.; Silva, N.B.; Castro, J.S.; Onety, J.A. 1990. Avaliação nutricional e alimentar de pré-escolares de uma creche de Manaus e a influência da entidade no estado nutricional de sua população.Acta Amazónica, 20:119-128.

NCHS, 1977. Growth curves children birth - 18, United States. Washington: National Center for Health Statistics. DC: U.S. Printing Office (Vital and Health Statistics Series 11, pub. $\mathrm{N}^{0} 78-1650$ ).

OMS. 1986. Use and interpretation of anthropometric indicators of nutritional status. Bull. World Health Organ., 64:929-41.

OMS. 1993. Situacion alimentaria y nutricional de America Latina. In: Conferencia Internacional sobre Nutricion. Santiago, Chile. OMS.

OPAS, 1994. Situación nutricional en las Americas. In: Boletin Epidemiológico. Organización Panamericana de la Salud OPAS. Vol. 15, $\mathrm{N}^{0} 3$, septiembre.

Pinheiro, M.F.S.; Vasconcelos, J.C.; Wendel, D.E. 1976. Contribuição ao estudo de parasitos intestinais em dois bairros de Manaus, Amazonas. Acta Amazônica, 6:67-73.

SEBRAE/AM, 1994. Diagnóstico sócioeconômico e cadastro empressarial de Barcelos. Manaus: Departamento de Estudo e Pesquisa, 199p.

Shrimpton, R.; Giugliano, R. 1979. Consumo de alimentos e alguns nutrientes em Manaus, Acta Amazónica, 9:117-141.

Shrimpton, R. 1984. Food consumption and dietary adequacy according to income in 1200 families, Manaus, Amazonas, Brazil, 1973-1974. Arch. Latenoamer Nutr. 34:615-629

Silva, M.V.; Pipitone, M.A. P.; Sturion, G. L.; Caroba, D. C. R. Educação, saúde e sua relação com o estado nutricional e praticas alimentares de escolares de $1^{\circ}$ grau. Anais do XVI Congresso Brasileiro de Ciencia e Tecnologia de Alimentos - Rio de Janeiro - SBCTA, 1998. v.1 pp 616-619.

Situação Mundial da Infầncia. 1995. Fundo das 
Nações Unidas para a Infância - UNICEF.

Trowbridge, F. L.; Newton, L.; Houng, A.; Stachling, N.; Valverde, V. 1980. Evaluacion de indicadores posa la vigilancia nutricional. Bol. Ofic. Sanit. Panamer., 89:589-95.

Vannucchi, H.; FREITAS, M.I.S.; SZARFARC, S.C. 1992. Prevalência de anemias nutricionais no Brasil. Cadernos de Nutrição. 4:7-26.

Vasconcelos, F.A.G-1993. Avaliação Nutricional de Coletividades: textos de apoio didático/ Francisco de Assis Guedes de Vasconcelos. Florianópolis: Editora do UFSC.

Waterlow, J.C.; Buzina, R.W.; Lane, J.M.; Machaman, M. Z. 1977. The presentation and use of height and weight data for comparing the nutritional status of grups of children under the age of 10 years. Bull. WHO, 55:189-98.

Yuyama, L. K. O.; Nagahama, D.; Marinho, H. A.; Vannucchi, H. 1989. Alimentação e estado nutricional de mães em diferentes estados fisiológicos de um bairro pobre de Manaus. Alimentos e Nutrrição, São Paulo. 1: 13-21. 